\title{
Many-to-Many Matching based Resource Allocation to support Multi-Connectivity
}

\author{
Haeyoung Lee ${ }^{1}$, Seiamak Vahid ${ }^{1}$, and Klaus Moessner ${ }^{2}$ \\ ${ }^{1}$ 5GIC, Institute for Communcation Systems (ICS), University of Surrey, U.K. \\ ${ }^{2}$ Professorship of Communications Engineering, Faculty of Electronics and information Technology, \\ University of Technology Chemnitz, Germany, and \\ 5GIC, Institute for Communication Systems, University of Surrey, UK. \\ Email: \{Haeyoung.Lee, S.Vahid, K.Moessner\}@ surrey.ac.uk
}

\begin{abstract}
In the fifth and beyond (5G/B5G) communication, wireless networks are evolved towards offering various services of different use cases and, therefore, need to span a wide range of requirements. While different services will be supported at the same time, radio resource management needs to consider the different requirements. In addition, as wireless systems are capable to support multi-connectivity, radio resource allocation becomes more challenging. In this context, we introduce a manyto-many matching game, and develop a distributed radio resource allocation algorithm supporting multi-connectivity. Simulation results demonstrate that the proposed approach improves the QoS levels of UEs by up to $14.9 \%$ considering their service requirements.
\end{abstract}

Index Terms-Multi-connectivity, utility-based QoS model, matching theory, many-to-many matching

\section{INTRODUCTION}

The fifth generation and beyond $(5 \mathrm{G} / \mathrm{B} 5 \mathrm{G})$ network will support high volumes of mobile data traffic and massive numbers of wireless connections. Different types of data (requiring different levels of Quality of Service (QoS)) are expected to be generated by various services. The major traffic types are enhanced mobile broadband (eMBB), massive machine type communication (mMTC), and ultra-reliable low latency communications (uRLLC) [1]. In order to meet the rapid growth in mobile data traffic and demands of wireless connectivity, wireless networks have evolved to dense and heterogeneous networks with different radio access technologies (RATs). As cells get denser and are overlapped, it is very likely that user equipments (UEs) would lie in the overlapping coverage of several base stations (BSs) or RATs, and multiple concurrent links would be available to UEs. In addition, more and more UEs are equipped with multiple radio interface and are capable of accessing multiple BSs or RATs. This UE apability and the availability of multiple links are geared towards enabling 5G applications with stringent QoS requirements through multiconnectivity $(M C)$ [2].

The initial goal of using $\mathrm{MC}$ was to boost data rate performance by splitting mobile data traffic and sending the traffic over multiple links, overcoming the capacity limitations constrained by backhaul links [3]. Mechanisms for traffic splitting/aggregation in multi-RAT heterogeneous networks (HetNets) have been investigated in literature. In [4], for a
HetNet consisting of a cellular macro BS and wireless LAN (WLAN) connected by non-ideal backhaul link, the optimal solution to split users' traffic across multiple links is proposed to maximise sum data rate. In [5], a joint user association and data rate allocation problem is solved to maximise total utility of data rate in a HetNet of multiple cellular BSs and WLAN access points. Similarly, the utility-based approach considering different data rate requirements of heterogeneous traffic types is studied in [6] for LTE and WLAN aggregation (LWA).

By adopting packet duplication (PD), multi-connectivity has been additionally considered as a approach to enhance reliability. In literature, the architecture enhancement in the radio access network (RAN) protocol stack [7], technical challenges to enable PD [8], and dynamic multi-connectivity configuration [3] are investigated for reliability improvement.

Whilst aforementioned studies [3]-[9] focus only on one type of service, $5 \mathrm{G}$ networks are supposed to provide various service types of different QoS requirements concurrently. In [10], multiple QoS parameters are dealt with for heterogeneous traffic types in a scenario where UEs can be associated with only one BS (single connectivity). In this paper, we consider multi-connectivity to support different service types. To effectively capture the levels of QoS provision of the service types, multiple QoS variables are considered. By using a utility-based approach, multiple QoS variables are integrated and the optimisation problem of radio resource allocation including the utility function is formulated. We apply a twosided many-to-many matching game model to the resource allocation problem and a novel algorithm for radio resource allocation is proposed. With the simulation results, we show that the proposed algorithm can support different types of service having different QoS requirements.

The remainder of the paper is organised as follows. In Section II, we describe the system model and the utilitybased QoS model integrating multiple QoS parameters for heterogeneous traffic types. In Section III, the resource allocation problem formulation is presented. Then, the proposed algorithm adopting many-to-many matching game is presented in Section IV. In Section V, we present the numerical analysis to validate the performance and efficiency of our proposed approach. Finally, conclusions are presented in Section VI. 


\section{System Model And Utility-BASed QoS Model}

\section{A. System Model}

In this paper, we consider the downlink transmission in $a b$ industrial network where a single operator (entity) owns its own macro BSs and small cell BSs and provides communication services for a geographic area. As shown in Fig. 1, a heterogeneous network consisting of multiple BSs can serve UEs with different service types. Let us consider a set of BSs $\mathcal{N}=\{1, \cdots, N\}$ where BS $n$ has a corresponding set of subchannels $\mathcal{K}=\{1, \cdots, K\}$ with a fixed bandwidth of $b_{n}^{k}$. There is also a set of user equipments (UEs) $\mathcal{M}=$ $\{1, \cdots, M\}$. Depending on UE's service type, different QoS parameters would be important. For instance, for uRLLC UEs, the core priority would be to ensure lower latency and bit error rate (BER) requirements. In order to effectively capture the required QoS level, three QoS parameters, data rate, BER, and delay, are considered and corresponding weight values are arranged. While UEs are executing an application of a specific service type, UEs are assumed to be able to associate with multiple BSs with the multi-connectivity capability. The set of BSs which UE $m$ is associated with is denoted as $\mathcal{A}_{m}$. Each BS can serve multiple UEs at the same time and the set of UEs associated with BS $n$ is denoted as $\mathcal{A}_{n}$. In order to decide UE's association and resource allocation accounting for QoS requirements, the utility-based approach described in Section II-B is adopted.

\section{B. Utility considering Multiple QoS Parameters}

To model the different QoS requirements of UEs, the utility function [10] including a set of three QoS parameters, data rate, BER and delay, is modelled as follows.

1) Data rate: We consider the data rate between $\mathrm{BS} n$ and UE $m$ via subchannel $k$ as the transmission capacity $C_{m n}^{k}$. It is defined using Shannon's capacity theorem, as follows.

$$
C_{m n}^{k}\left(b_{m n}^{k}\right)=b_{m n}^{k} \log \left(1+\gamma_{m n}^{k}\right) .
$$

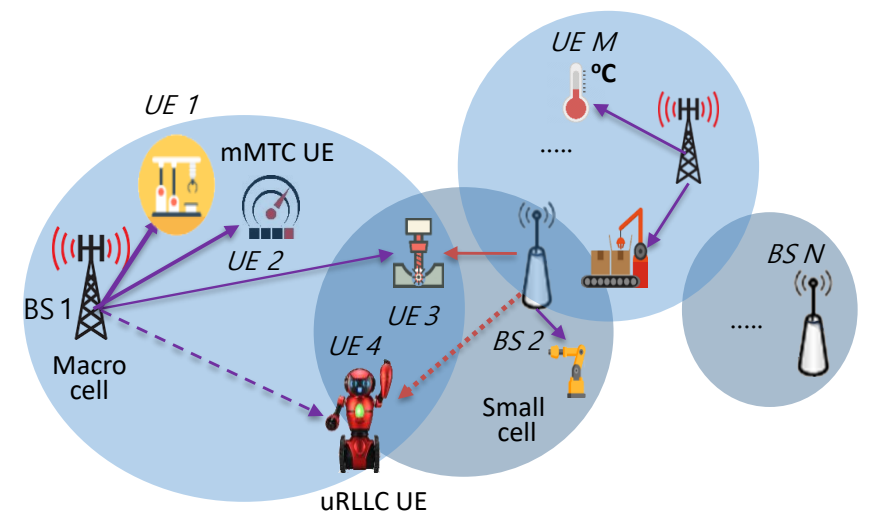

Fig. 1. Downlink transmission to UEs with different service types through multi-connectivity in a HetNet
In (1), $b_{m n}^{k}$ denotes the bandwidth of subchannel $k$ of BS $n$ which is allocated to UE $m$ and the signal-to-interferenceplus-noise ratio (SINR) $\gamma_{m n}^{k}$ can be calculated as follows.

$$
\gamma_{m n}^{k}=\frac{p_{m n}^{k} g_{m n}^{k}}{I_{m n}^{k}+\sigma^{2}},
$$

where $p_{m n}^{k}$ is the transmit power and $g_{m n}^{k}$ is the channel gain between BS $n$ and UE $m$ via subchannel $k$ which accounts for path loss and fading. $\sigma^{2}$ denotes the variance of the Additive White Gaussian Noise (AWGN). As the channel interference term, $I_{m n}^{k}$ indicates interference from other BSs $n^{\prime}$, i.e., $I_{m n}^{k}=$ $\sum_{n^{\prime} \neq n} p_{n^{\prime}}^{k} g_{n^{\prime}}^{k}$ where $1 \leq n^{\prime} \leq N . p_{n^{\prime}}^{k}$ and $g_{n^{\prime}}^{k}$ are the transmit power and channel gain of transmission between BS $n^{\prime}$ and their associated UEs via the subchannel $k$, respectively.

2) BER: The transmitted data between BS $n$ and UE $m$ could be corrupted depending on the channel conditions. We consider the BER model derived in [11] as follows.

$$
E_{m n}^{k}= \begin{cases}0.2 \times \exp \left(\frac{-1.6 \times \gamma_{m n}^{k}}{2^{h\left(\gamma_{m n}^{k}\right)}-1}\right) & \text { for } \gamma_{m n}^{k} \geq \gamma_{t h}, \\ 1 & \text { for otherwise. }\end{cases}
$$

In (3), $h\left(\gamma_{m n}^{k}\right)$ is the modulation efficiency of a given modulation scheme and $\gamma_{t h}$ is the threshold of the modulation scheme.

3) Delay: For delay performance, transmission delay and queuing delay are considered. Whilst the transmission delay is determined by the amount of time to transmit $N_{t x}$ bits via the link at data rate $C_{m n}^{k}\left(b_{m n}^{k}\right)$, the queuing delay is affected by the congestion level of BS $n$. The data packet transmission process at each $\mathrm{BS}$ is modelled as a $M / M / 1$ queueing system [12]. It is assumed that the mean arrival traffic rate and service rate of BS $n$ are $\lambda_{n}$ (packets/sec) and $\mu_{n}$ (packets/sec), respectively, in the slow fading channels. Note for simplicity, the retransmission of the packets which are erroneously received is not considered. In addition, the stable case of $\lambda_{n} / \mu_{n}<1$ is considered. The average delay for the traffic of UE $m \in \mathcal{A}_{n}$ can be defined as a sum of transmission delay and queuing delay [12] and derived as follows.

$$
D_{m n}^{k}=\frac{N_{t x}}{C_{m n}^{k}\left(b_{m n}^{k}\right)}+\frac{1}{\mu_{n}-\lambda_{n}} .
$$

4) Context-aware utility function of multiple QoS parameters: The above QoS parameters can be integrated into a utility function to express the satisfaction level for the required QoS. As can be seen in (5), three parameters $C_{m n}^{k}, E_{m n}^{k}$, and $D_{m n}^{k}$, are weighted with respect to their relative significance in QoS provision for different service types. The corresponding weighting values are denoted as $w_{m}^{C}, w_{m}^{E}$, and $w_{m}^{D}$, respectively. A set of weight values $\mathcal{W}_{m}=\left\{w_{m}^{C}, w_{m}^{E}, w_{m}^{D}\right\}$ is assumed to be given for UE $m$ considering QoS requirements. While a set of weight values can be different for different services, the sum of all weights always equals to 1 . The context-aware utility function [10] can be defined as a weighted product of three QoS parameters as follows.

$$
U_{m n}^{k}\left(b_{m n}^{k}, \mathcal{W}_{m}\right)=\frac{w_{m}^{C} C_{m n}^{k}\left(b_{n}^{k}\right) \cdot w_{m}^{E}\left(1-E_{m n}^{k}\right)}{w_{m}^{D} D_{m n}^{k}} .
$$


5) Utility function for multi-connectivity: Whilst the utility $U_{m n}^{k}$ in (5) indicates the utility value achievable from the single connectivity between BS $n$ and UE $m$, the utility value will be affected by the multi-connectivity. For the purpose of increasing data rate, when a UE is connected to multiple BSs, the different data is transmitted over multiple links and aggregated. For the reliability enhancement purpose, the same data is transmitted across multiple links. Then, the first arriving data is processed whereas the late arriving duplicate is discarded [8]. In this paper, the utility of UE $m$ achievable from the multi-connectivity is defined as the sum of utility of links with BSs $n \in \mathcal{A}_{m}$ as follows.

$$
U_{m} \triangleq \sum_{n \in \mathcal{A}_{m}} U_{m n}\left(b_{m n}, \mathcal{W}_{m}\right)
$$

\section{PRoblem Formulation}

The goal of user association and resource allocation strategy is set to maximize the satisfaction level of QoS requirements of UEs. The problem can be formulated as follows.

$$
\begin{array}{ll}
P: & \max _{\alpha_{m n}, \delta_{m n}^{k}} \sum_{m \leq M} U_{m}\left(\alpha_{m n}, \delta_{m n}^{k}, b_{m n}, \mathcal{W}_{m}\right), \\
\text { s.t. } & \alpha_{m n} \delta_{m n}^{k} C_{m n}^{k}\left(b_{m n}^{k}\right) \geq C_{m}^{\text {req }}, \quad \forall m \leq \mathcal{M}, \\
& E_{m n}^{k}\left(\alpha_{m n}, \delta_{m n}^{k}\right) \leq E_{m}^{\text {req }}, \quad \forall m \in \mathcal{M}, \\
& D_{m n}^{k}\left(\alpha_{m n}, \delta_{m n}^{k}\right) \leq D_{m}^{\text {req }}, \quad \forall m \in \mathcal{M}, \\
& w_{m}^{C}+w_{m}^{E}+w_{m}^{D}=1, \quad \forall m \in \mathcal{M} . \\
& \alpha_{m n}, \delta_{m n}^{k}=\{0,1\}, \quad \forall m \in \mathcal{M}, \forall n \in \mathcal{N}, \forall k \in \mathcal{K}, \\
& \sum_{m \leq M} \delta_{m n}^{k} \leq 1, \quad \forall k \in \mathcal{K}, \\
& \sum_{m \leq M} \alpha_{m n} \leq q_{n}, \quad \forall n \in \mathcal{N}, \\
& \sum_{n \leq N} \alpha_{m n} \leq q_{m}, \\
&
\end{array}
$$

The objective function in (7) is formulated with the aim to maximise the sum of the utility value of each UE $m$. The constraints in (8)-(10) describe the QoS requirements of UE $m$ where $C_{m}^{\text {req }}, E_{m}^{\text {req }}$ and $D_{m}^{\text {req }}$ are the requirements of data rate, BER, and delay, respectively. Whilst the weight value for each QoS parameter can be set differently depending on the service type, (11) refers that the sum of weights equals to 1. In (12), $\alpha_{m n}$ is a binary allocation indicator where $\alpha_{m n}$ equals to 1 if UE $m$ is associated with BS $n$, and 0 otherwise. Similarly, $\delta_{m n}^{k}$ becomes 1 if subchannel $k$ is selected by BS $n$ for UE $m$, and 0 otherwise. The constraint in (13) addresses that BS $n$ can allocate subchannel $k$ exclusively to one UE. In (14), the constraint indicates BS $n$ can associate with a limited number of UEs and $q_{n}$ is the quota value. Since it is assumed that one subchannel is allocated to each UE, $q_{n}$ becomes equal to the number of subchannels of BS $n$. The constraint in (15) refers to multi-connectivity capability of UE $m$. In the dualconnectivity case, $q_{m}$ equals to 2 . If UE $m$ is capable of being associated with only one BS, $q_{m}$ becomes 1 .

The problem formulated in (7) can be compared to a $0 / 1$ multiple-knapsack problem with the constraints in (8)-(15).
Similar to the $0 / 1$ multiple-knapsack problem which is a wellknown NP hard combinatorial optimisation problem [13], the combinatorial nature of the problem in (7) leads to find all the feasible allocations where its complexity is $\mathcal{O}\left(2^{M \times N \times K}\right)$ [10]. For a large size network with a very large number of BSs, subchannels, and UEs, the complexity increases exponentially, thus the optimisation problem becomes intractable. Therefore, we adopt the matching game theory that can provide low complexity distributed solutions to the combinatorial problem. In the next section, the matching based approach is explained.

\section{Context Aware Resource allocation by using MATCHING THEORY}

\section{A. The Matching Game Formation}

To model the resource allocation approach as a many-tomany matching game [14], we consider two sets of UEs $\mathcal{M}$ and BSs $\mathcal{N}$ as two teams of players with $\mathcal{M} \cap \mathcal{N}=\phi$. The matching is defined as an assignment of UEs in $\mathcal{M}$ to BSs in $\mathcal{N}$. Since a UE can be assigned to multiple BSs and an BS can accept multiple UEs, the considered game becomes a type of a many-to-many matching game. While each player (UEs and BSs) is assumed to behave independently, this matching game can operate in a distributed way. In a matching game, the quota of a player is defined as the maximum number of players in the opposite team that the player can be matched to. In our scenario, UE $m$ has a quota $q_{m}$ such that UE $m$ can be associated with at most $q_{m}$ BSs with the multiconnectivity capability. In turn, BS $n$ has a quota $q_{n}$ indicating that BS $n$ can accommodate $q_{n}$ numbers of UEs. In matching theory, each player tends to seek the most preferred partners for matching. The outcome of the matching game is a matching function $\pi$ and this is defined as the follows.

Definition 1. A many-to-many matching $\pi$ is defined to mapping each player $m \in \mathcal{M}$ to $n \in \mathcal{N}$ under the following conditions of the matching $\pi: \mathcal{M} \cup \mathcal{N} \Longrightarrow 2^{\mathcal{M} \cup \mathcal{N}}$ such that,

1) $\pi(m) \subseteq \mathcal{N}$ such that $|\pi(m)| \leq q_{m}, \forall m \in \mathcal{M}, \forall n \in \mathcal{N}$,

2) $\pi(n) \subseteq \mathcal{M}$ such that $|\pi(n)| \leq q_{n}, \forall m, \forall n$,

3) $n \in \pi(m)$ if and only if $m \in \pi(n), \forall m, \forall n$,

In condition 1) and 2), $\pi(m)$ and $\pi(n)$ refer to the set of player $m$ 's and $n$ 's matched partners, respectively, under the matching relation $\pi \cdot|\pi(\cdot)|$ indicates the cardinality of $\pi(\cdot)$. As per (14) and (15), $q_{m}$ and $q_{n}$ is the maximum capacity of $m \in \mathcal{M}$ and $n \in \mathcal{N}$, respectively. Condition 3) states that if BS $n$ is matched to UE $m$, UE $m$ is also matched to BS $n$, which is naturally given in a UE-BS association problem. The matched pair can be denoted as $(m, n)$.

In matching, each player defines its preference over players in the opposite team. Whilst the preference relation is denoted as $\succ$, the expression $m \succ_{n} m^{\prime}$ implies that UE $n$ prefers to be associated with BS $m$ rather than BS $m^{\prime}$ (where $m \neq m^{\prime}$ ). A similar notation is used for BSs to indicate their preference. The level of preference is managed with a preference list as per Definition 2. 
Definition 2. A preference list of $m \in \mathcal{M}$ or $n \in \mathcal{N}$ is an ordered set including all the possible players in the opposite set $\mathcal{N}$ or $\mathcal{M}$. The preference relation in the preference lists hold the transitive property within the matching game, i.e., if for $n \succ_{m} n^{\prime}$ and $n^{\prime} \succ_{m} n^{\prime \prime}$, we have $n \succ_{m} n^{\prime \prime}$, where $m$ is a player of matching game and $n, n^{\prime}$, and $n^{\prime \prime}$ are the subsets of player in $m$ 's opposite set.

The preference list of $m$ and $n$ are denoted as $p_{m}$ and $p_{n}$, respectively. To decide the preference list of UE $m$, the expected utility is considered as,

$$
\bar{U}_{m n}=w_{m}^{C} C_{m n}\left(b_{n}\right) \cdot w_{m}^{E}\left(1-E_{m n}\right) .
$$

The expected utility function in (16) includes the data rate and BER parameters $C_{m n}$ and $E_{m n}$ with the respective weight factors $w_{m}^{C}$ an $w_{m}^{E}$. It indicates the attainable level of satisfaction of UE $m$ associated with BS $n$. In its preference list $p_{m}$, the preference relation can be expressed as,

$$
n \succ_{m} n^{\prime} \Leftrightarrow \bar{U}_{m n}>\bar{U}_{m n^{\prime}}, \quad \text { where } n \neq n^{\prime} .
$$

(17) refers that $m$ prefers $n$ to $n^{\prime}$ as the expected utility $\bar{U}_{m n}$ is higher than $\bar{U}_{m n^{\prime}}$.

Similarly, BSs determine their preference on UEs. While BSs are assumed to prefer to increase the spectral efficiency to provide communication connectivity, the level of reference signal receive power (RSRP) based on measurement report feedback by UEs can be taken into account for deciding BSs' preference on UEs. In the preference list $p_{n}$ of $\mathrm{BS} n$, the rank or preference $\succ_{n}$ is given as,

$$
m \succ_{n} m^{\prime} \Leftrightarrow R S R P_{m}>R S R P_{m^{\prime}} \text {, where } m \neq m^{\prime} .
$$

In (18), whilst the RSRP level received by UE $m$ is larger than the RSRP by $m^{\prime}$, BS $n$ prefers UE $m$ more than $m^{\prime}$ and these relationships are included in $p_{n}$.

Our matching game exhibits externalities indicating interdependencies between the players' preferences. Regarding the data rate in (1), BER in (3) and the delay in (4), they could be affected by the channel interference and traffic loads. The player's preference depends not only on the information available at this player, but also on the others' matchings. Due to externalities, a player may keep changing its preference order, in response to the formation of other UE-BS mapping. Since the matching will never reach a final state, it is important to handle externalities carefully [14].

A matching is called as 'stable' if no players obtain any gains from further changing their matching. A stable matching would not be blocked by an individual or pair(s) of players, who mutually prefer each other to their respective partners [15]. We introduce the concept of blocking pair to describe BSs' decision about UE's proposal and defined as follows.

Definition 3. For a given matching $\pi$ where $n \in p_{m}$ and $m \in p_{n}$ and its pairs $\left(m, n^{\prime}\right)$ and $\left(m^{\prime}, n\right),(m, n)$ is a blocking pair if $n \notin \pi(m)$, and $n \succ_{m} n^{\prime}, n \notin \pi(m)$, and $m \succ_{n} m^{\prime}$.

The blocking pair $(m, n)$ is considered only when $m$ and $n$ have not matched with each other and $n$ is in the preference list of $m$ and $m$ is in the preference list of $n$. In addition, matching of $m$ and $n$ can increase the interest of both $m$ and $n$, representing the current matching formation is not optimal. With the definition of blocking pair, we explain how BSs decide whether to accept UEs' proposal. The following three cases can be considered from BS $n$.

(A) $\tilde{C}_{n}\left(\tilde{b}_{n}\right) \geq C_{m}^{\text {req }}, m \succ_{n} \phi$ and $n \succ_{m} n^{\prime}$,

(B) $\tilde{C}_{n}\left(\tilde{b}_{n}\right)<C_{m}^{\text {req }}, \tilde{C}_{n}+\sum_{m^{\prime} \in \mathcal{A}_{n}} C_{m^{\prime} n}\left(b_{m^{\prime} n}\right) \geq C_{m}^{\text {req }}$, where $m \succ_{n} m^{\prime}$ and $n \succ_{m} n^{\prime}$,

(C) $U_{m n}<\bar{U}_{m n}, n \succ_{m} n^{\prime}$ and $m \succ_{n} m^{\prime}$.

In (A), if BS $n$ has residual quota $\tilde{C}_{n}\left(\tilde{b}_{n}\right)=C_{n}\left(b_{n}\right)-$ $\sum_{m^{\prime} \in \mathcal{A}_{n}} C_{m^{\prime} n}\left(b_{m^{\prime} n}\right)$ enough to accommodate UE $m$ and UE $m$ prefers BS $n$ over its current association with $\mathrm{BS} n^{\prime}$, BS $n$ accepts the proposal from UE $m$. Due to UEs $m^{\prime}$ already being attached to BS $n$, the residual quota $\tilde{C}_{n}\left(\tilde{b}_{n}\right)$ of BS $n$ would not be sufficient to accommodate UE $m \in p_{n}$ as mentioned in (B). Then, BS $n$ rejects the least preferred $\mathrm{UE} m^{\prime} \in \mathcal{A}_{n}$ and admits the proposal from $m$. In (C), a challenge stemming from peer effects is considered. The matching utility $U_{m n}$ between UE $m$ and BS $n$ defined in (5) is less than the expected utility $\bar{U}_{m n}$ in (16). It indicates the performance degradation due to peer effects. In such case, UE $m$ and BS $n$ deviate from their current associations and become a matched pair. In our matching problem, the preference lists of UEs are correlated with the matching formation of UEs and BSs due to the peer effects. Hence, the static preference list becomes no longer appropriate. We consider dynamically updating the preference lists of UEs according to the matching resulted from each static matching process. The matching process will be repeated until all UEs are completely matched or the preference lists of UEs become empty. To decrease our algorithm' complexity, repeated attempts of formerly rejected proposals should be prevented. To exclude the rejecting BSs from the preference list of UEs, we introduce the concept of a rejection list. When $n$ rejects $m, n$ is added into the rejection list of UE $m, \mathcal{R}_{m}$.

\section{B. The Proposed Algorithm}

Our matching based resource allocation algorithm is presented in Algorithm 1. First, the preference lists $p_{m}$ and $p_{n}$ of UE $m$ and BS $n$ are calculated. The list of accepted UEs by BS $n, \mathcal{A}_{n}$, the list of BSs accepting UE $m, \mathcal{A}_{m}$ and the rejection list of UE $m, \mathcal{R}_{m}$ are initialised. In the algorithm, the matching process works by applying successively the following procedure. At each iteration $t$, each UE $m$ starts making proposals to its most preferred BS(s) included in its preference list $p_{m}$ but not included in the rejection list $\mathcal{R}_{m}$. After UE's proposal, the indices of selected $n$ are deleted from UE's preference list $p_{m}$ (lines 6-8). While the proposal from UE $m$ is received by BS $n$, BS $n$ considers the proposal only from $m$ appeared in $p_{n}$. When BS $n$ has the capability to accommodate UE $m$, BS $n$ considers to hold its proposal. In this case, the quota of UE $m, q_{m}$ is considered. When the number of BSs which UE $m$ is matched with is less than $q_{m}$, BS $n$ holds the proposal from UE $m$ (lines 1112). When UE $m$ has already matched with BSs, UE $m$ 


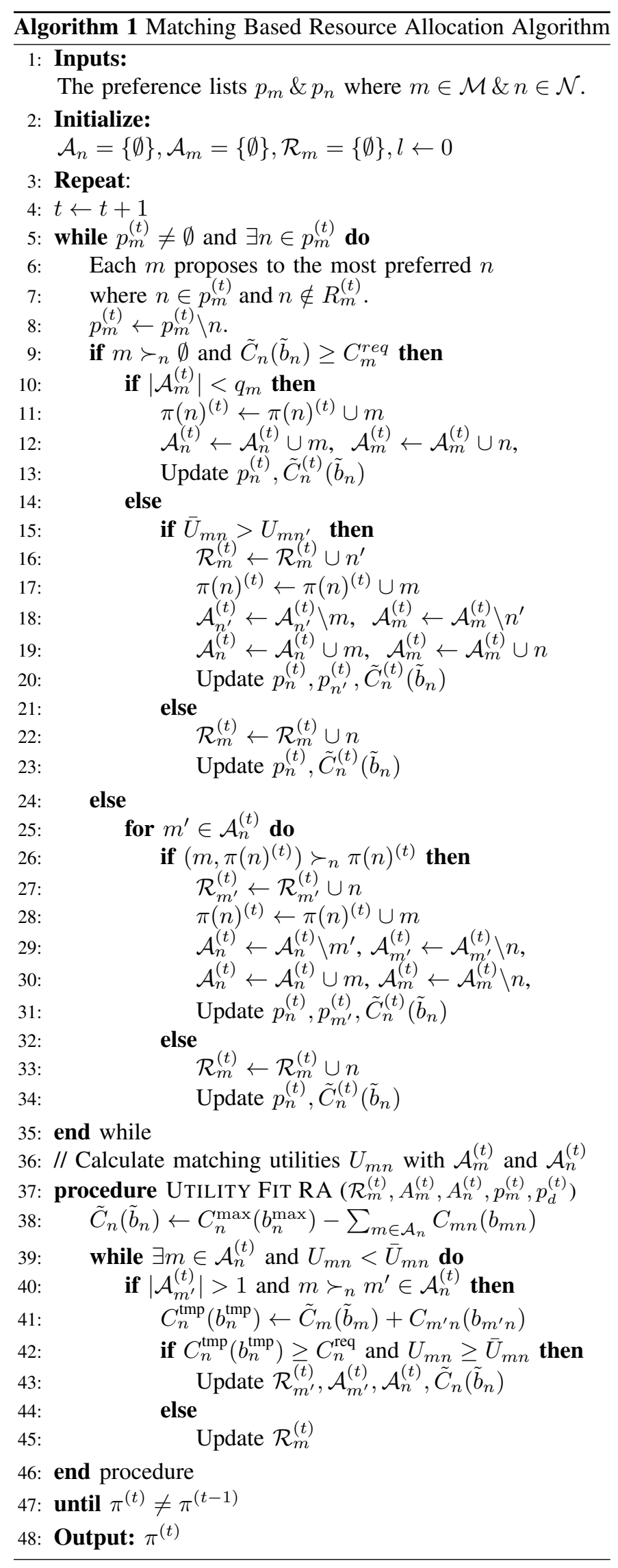

TABLE I

SIMULATION PARAMETERS

\begin{tabular}{|l|c|}
\hline Parameters & Values \\
\hline \hline Layout & $100 \mathrm{~m} \mathrm{x} 100 \mathrm{~m} \mathrm{x} 10 \mathrm{~m}$ \\
\hline No. BS \& Tx pwr & $1 \mathrm{MBS}(5 \mathrm{dBm}), 2 \mathrm{SBSs}(0 \mathrm{dBm})$ \\
\hline Thermal noise & $-174 \mathrm{dBm} / \mathrm{Hz}$ \\
\hline System bandwidth & $10 \mathrm{MHz} @ 3.5 \mathrm{GHz}$ \\
\hline Height of BS/UE & $3 \mathrm{~m} / 1 \mathrm{~m}$ \\
\hline No. UEs & varying in [20-60], mMTC \& uRLLC UEs \\
\hline UE packet size & 50 bytes (uRLLC UEs), 480 bytes $(\mathrm{mMTC}$ UEs) \\
\hline Path Loss & $\begin{array}{c}\text { LOS: } 32.45+20 \log \left(d_{3 d}\right)+20 \log (f)+X_{\sigma_{1}}, \\
\text { nLOS: } 32.45+24.7 \log \left(d_{3 d}\right)+20 \log (f)+X_{\sigma_{2}}, \\
\text { where } \sigma_{1}=3 \mathrm{~dB}, \sigma_{2}=5.17 \mathrm{~dB}, d_{3 d} \geq 1 \mathrm{~m}\end{array}$ \\
\hline
\end{tabular}

compares the utility $U_{m n^{\prime}}$ of the existing matching $\pi\left(m, n^{\prime}\right)$ with the expected matching utility $\bar{U}_{m n}$ of $\pi(m, n)$ (line 15). If the expected utility of $\pi(m, n)$ is higher than the utility of the prior matching $\pi\left(m, n^{\prime}\right)$, UE $m$ puts BS $n^{\prime}$ into its rejection list $\mathcal{R}_{m}$ and a new matching $\pi(m, n)$ is created. Consequently, BS $n^{\prime}$ removes the matching $\pi\left(m, n^{\prime}\right)$ from its accepted list $\mathcal{A}_{n^{\prime}}$ and BS $n$ adds the matching $\pi(m, n)$ to its accepted list $\mathcal{A}_{n}$ (lines 16-19). Otherwise, UE $m$ keeps the existing matches and stores BS $n$ in the rejection list $\mathcal{R}_{m}$. If $\mathrm{BS} n$ is not capable to accommodate the new proposal from UE $m, n$ finds any least favourable matching instance from the accepted list $\mathcal{A}_{n}$. If there is any least favourable matching $\pi\left(m^{\prime}, n\right)$, BS $n$ rejects and removes the existing matching $\pi\left(m^{\prime}, n\right)$ from $\mathcal{A}_{n}$ and accepts the proposal from $m$. Then, the new matching $\pi(m, n)$ will be created (lines 2730). In case that any matching instances are created or rejected, the corresponding preference lists, rejection lists, and residual capacity of BS $m$ are updated accordingly (line 13, 20, 23, 31, and 34). Whilst the proposed algorithm is to solve the problem in (7) including context-based QoS requirements in (8)-(10), the utility $U_{m n}$ would be affected by other UE's matchings. To manage the effect of externalities, similarly to [10], we consider the utility fitting procedure ensuring the convergence and stability of the matching game as presented (lines 37-46).

\section{Simulation RESUlts}

We evaluate the performance of the proposed algorithm via simulation. Table I shows the configuration parameters. More parameters about traffic and channel models are found in [16].

First, we compare the average utility values of UEs requesting different types of traffic. For the reference scheme (labelled 'Random'), random allocation which does not consider any channel and traffic parameters is selected. Fig. 2 shows that the proposed approach considering channel quality and traffic specific characteristics can exploit the radio resource more efficiently by producing higher average utility values.

In Fig. 3 and Fig. 4, BER and delay performance are shown, respectively. For the reference scheme (labelled 'Reference2'), the approach considering SINR [15] is chosen. Whilst BER and delay QoS parameters are important for uRLLC traffics, it is shown that the proposed approach can support the QoS of URLLC traffics better than the reference scheme. The BER values in Fig. 3 can be converted to the BLER values [17]. 


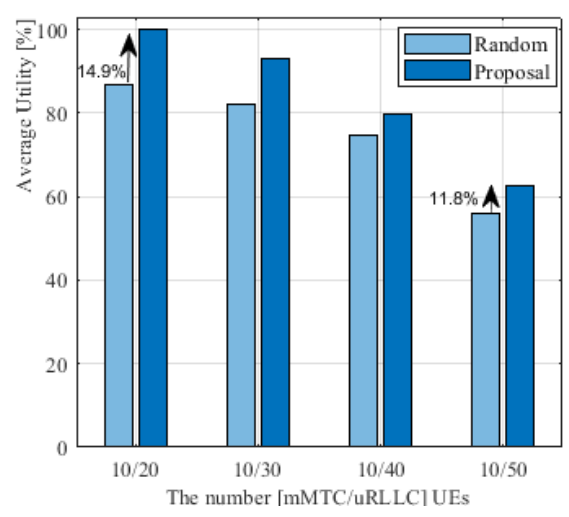

Fig. 2. Comparison of average utility values

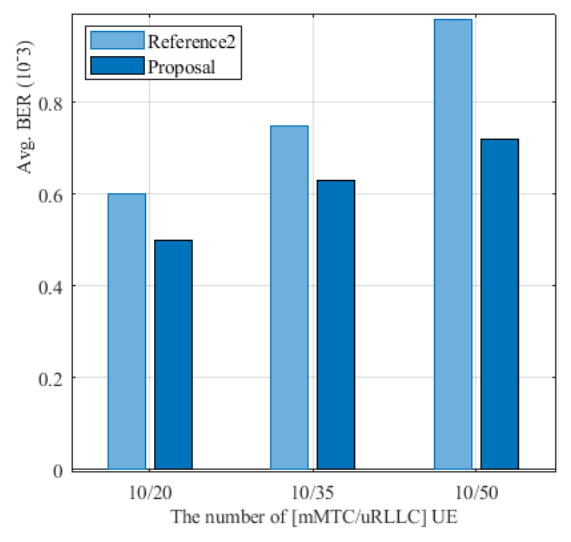

Fig. 3. BER Performance comparison

Then, the BLER values become [0.0291, 0.368, 0.0433] for the 'Reference2' scheme whilst BLER are [0.0362, 0.0468, 0.0585] for the 'Proposal' scheme. The proposed scheme improves the BLER performance by $20-25 \%$ compared to the reference scheme. In terms of delay performance, the proposed algorithm outperforms the reference approach by around 23$48 \%$ depending on the different numbers of UEs.

\section{Conclusions}

In this paper, investigations into resource allocation supporting multi-connectivity for heterogeneous traffic was provided. Whilst multiple QoS parameters are integrated in a utility function, two-sided many-to-many matching game approach is adopted in the proposed algorithm. By the simulation results, the proposed algorithm is shown to improves QoS level of UEs by up to $14.9 \%$ considering their achieved service requirements even. In future research, we will consider the effect of non-ideal backhaul on the performance, especially throughput and delay. In addition, more comprehensive analysis using the system level simulations will be conducted.

\section{ACKNOWLEDGMENT}

This work has been performed as a part of the CLEAR5G project supported by the EU H2020 under grant agreement no.

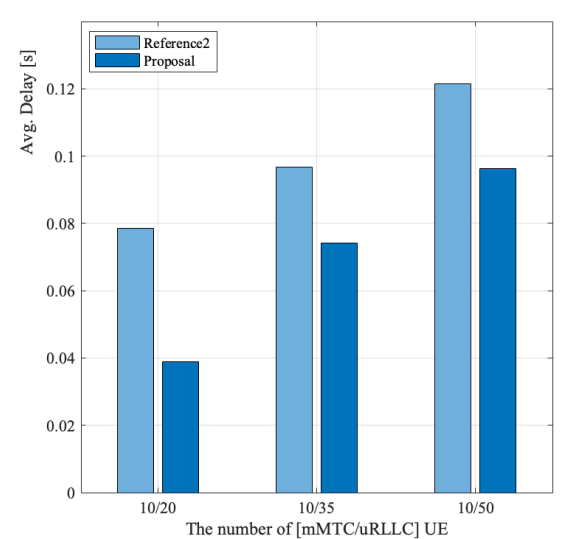

Fig. 4. Delay Performance comparison

761745 and the Government of Taiwan. Authors acknowledge 5 GIC at the University of Surrey.

\section{REFERENCES}

[1] Nokia Networks, "5G use cases and requirements ," July 2014.

[2] 3GPP, "Evolved Universal Terrestrial Radio Access (E-UTRA) and NR; Multi-connectivity; Stage 2," TS 37.340, V15.6.0, June 2019.

[3] H. Lee, S. Vahid, and K. Moessner, "Machine Learning Based RATs Selection Supporting Multi-connectivity for Reliability," in Proc. EAI Conf. Cognitive Radio-Oriented Wireless Netw. (CrownCom), June 2019.

[4] S. Singh et al., "Proportional Fair Traffic Splitting and Aggregation in Heterogeneous Wireless Networks," IEEE Commun. Lett., vol. 20, no. 5, pp. 1010-1013, May 2016.

[5] A. R. Ekti et al., "Joint User Association and Data-Rate Allocation in Heterogeneous Wireless Networks," IEEE Trans. Veh. Technol., vol. 65, no. 9 , pp. 7403-7414, Sep. 2016.

[6] H. Lee, S. Vahid, and K. Moessner, "Traffic-Aware Resource Allocation with Aggregation in Heterogeneous Networks with WLANs," in Proc. 2018 European Conf. Netw. Commun. (EuCNC), June 2018.

[7] J. Rao and S. Vrzic, "Packet Duplication for URLLC in 5G: Architectural Enhancements and Performance Analysis," IEEE Netw., vol. 32, no. 2, pp. 32-40, March 2018.

[8] A. Aijaz, "Packet Duplication in Dual Connectivity Enabled 5G Wireless Networks: Overview and Challenges," IEEE Commun. Standards Mag., vol. 3, no. 3, pp. 20-28, Sep. 2019.

[9] Y. Wu, Y. He, L. P. Qian, J. Huang, and X. Shen, "Optimal Resource Allocations for Mobile Data Offloading via Dual-Connectivity," IEEE Trans. Mobile Comput., vol. 17, no. 10, pp. 2349-2365, Oct. 2018.

[10] S. F. Abedin et al., "Resource Allocation for Ultra-Reliable and Enhanced Mobile Broadband IoT Applications in Fog Network," IEEE Trans. Commun., vol. 67, no. 1, pp. 489-502, Jan. 2019.

[11] S. T. Chung and A. J. Goldsmith, "Degrees of freedom in adaptive modulation: a unified view," IEEE Trans. Commun., vol. 49, no. 9, pp. 1561-1571, Sept. 2001.

[12] Gnedenko, B. V et al., Introduction to Queuing Theory, 2nd ed., ser. Mathematical Modeling. Boston, MA: Birkhäuser Boston, 1989, vol. 5.

[13] S. R. Balachandar and K. Kannan, "A new polynomial time algorithm for 0-1 multiple knapsack problem based on dominant principles," Applied Mathematics and Computation, vol. 202, no. 1, pp. 71-77, 2008.

[14] Y. Gu et al., "Matching Theory for Future Wireless Networks: Fundamentals and Applications," IEEE Commun. Mag., vol. 53, no. 5, pp. 52-59, May 2015.

[15] M. Simsek, T. Hößler, E. Jorswieck, H. Klessig, and G. Fettweis, "Multiconnectivity in Multicellular, Multiuser Systems: A MatchingBased Approach," Proc. IEEE, vol. 107, no. 2, pp. 394-413, Feb. 2019.

[16] CLEAR5G, "Deliverable D1.1: System Specifications and Business Perspectives, v1.1," Feb. 2019.

[17] M. Hamza et al., "BER-based BLER prediction for LTE FDD DL channel with small delay dispersion," in Prof. 2015 Int'l Wireless Commun, Mobile Comput. Conf. (IWCMC), Aug. 2015, pp. 1156-1161. 